\title{
A New Method for Proving Existence Theorems for Abstract Hammerstein Equations
}

\author{
C. E. Chidume, ${ }^{1}$ C. O. Chidume, ${ }^{2}$ and Ma'aruf Shehu Minjibir ${ }^{1,3}$ \\ ${ }^{1}$ African University of Science and Technology, Abuja 900241, Nigeria \\ ${ }^{2}$ Department of Mathematics and Statistics, Auburn University, Auburn, AL 36849-5168, USA \\ ${ }^{3}$ Department of Mathematical Sciences, Bayero University, Kano 700241, Nigeria
}

Correspondence should be addressed to C. E. Chidume; cchidume@aust.edu.ng

Received 11 December 2014; Accepted 23 February 2015

Academic Editor: Simeon Reich

Copyright (C) 2015 C. E. Chidume et al. This is an open access article distributed under the Creative Commons Attribution License, which permits unrestricted use, distribution, and reproduction in any medium, provided the original work is properly cited.

An abstract Hammerstein equation is an equation of the form $u+K F u=0$. A new method is introduced to prove the existence of a solution of this equation where $K$ and $F$ are nonlinear accretive (monotone) operators. The method does not involve the complicated technique of factorizing a linear map via a Hilbert space and does not involve the use of deep variational techniques.

\section{General Introduction}

Let $E$ be a real normed space and let $S:=\{x \in E:\|x\|=1\}$. The space $E$ is said to have Gâteaux differentiable norm if the limit

$$
\lim _{t \rightarrow 0} \frac{\|x+t y\|-\|x\|}{t}
$$

exists for all $x, y \in S$; in this case $E$ is said to be smooth. $E$ is said to have uniformly Gâteaux differentiable norm if, for each $y \in S$, the limit is attained uniformly for $x \in S$. Further, $E$ is said to be uniformly smooth if the limit is attained uniformly for $(x, y) \in S \times S$. The modulus of smoothness of $E$, $\rho_{E}:[0, \infty) \rightarrow[0, \infty)$, is defined by

$$
\begin{array}{r}
\rho_{E}(\tau) \\
:=\sup \left\{\frac{\|x+y\|+\|x-y\|}{2}-1:\|x\|=1,\|y\|=\tau\right\} ; \\
\tau>0 .
\end{array}
$$

$E$ is equivalently said to be smooth if $\rho_{E}(\tau)>0 \forall \tau>0$. Let $q>1 ; E$ is said to be $q$-uniformly smooth (or to have a modulus of smoothness of power type $q$ ) if there exists $c>0$ such that $\rho_{E}(\tau) \leq c \tau^{q}$.

$L_{p}, l_{p}$, and the Sobolev space $W_{m}^{p}, 1<p<\infty$, are all $q$-uniformly smooth. In fact

$$
L_{p} \text { or } l_{p} \text { or } W_{m}^{p} \text { is } \begin{cases}p \text {-uniformly smooth, } & 1<p \leq 2, \\ 2 \text {-uniformly smooth, } & p \geq 2 .\end{cases}
$$

Furthermore (see, e.g., [1]),

$$
\begin{aligned}
& \rho_{L_{p}}(\tau) \\
& =\rho_{l_{p}}(\tau)=\rho_{W_{m}^{p}}(\tau) \\
& \quad= \begin{cases}\left(1+\tau^{p}\right)^{1 / p}-1<\frac{1}{p} \tau^{p}, & 1<p \leq 2, \\
\frac{(p-1)}{2} \tau^{2}+o\left(\tau^{2}\right)<\frac{p-1}{2} \tau^{2}, & p \geq 2 .\end{cases}
\end{aligned}
$$

Let $J_{q}$ denote the generalized duality mapping from $E$ to $2^{E^{*}}$ defined by

$$
J_{q}(x):=\left\{f \in E^{*}:\langle x, f\rangle=\|x\|^{q},\|f\|=\|x\|^{q-1}\right\},
$$


where $E^{*}$ denotes the dual space of $E$ and $\langle\cdot, \cdot\rangle$ denotes the generalized duality pairing. It is well known (see, e.g., $\mathrm{Xu}$ [2]) that $J_{q}(x)=\|x\|^{q-2} J(x)$ if $x \neq 0$ where $J$ denotes $J_{2}$ (called the normalized duality mapping). It is well known that if $E^{*}$ is strictly convex, $J$ is single-valued. For more information and examples concerning (generalized) duality mappings, one may see the book of Cioranescu [3] and its review by Reich [4]. In the sequel, we will denote the singlevalued duality map by $j$.

A map $A: D(A) \subset X \rightarrow X$ is called accretive if, for all $x, y \in D(A)$, there exists $j(x-y) \in J(x-y)$ such that the following inequality holds:

$$
\langle A x-A y, j(x-y)\rangle \geq 0 .
$$

If $X$ is a real Hilbert space, the map $A$ is called monotone. In this case, $A$ satisfies the following condition:

$$
\langle A x-A y, x-y\rangle \geq 0 .
$$

The map $A$ is called strongly accretive if there exists $c>0$ such that, for all $x, y \in D(A)$, there exists $j(x-y) \in J(x-y)$, such that

$$
\langle A x-A y, j(x-y)\rangle \geq c\|x-y\|^{2} .
$$

A nonlinear integral equation of Hammerstein type (see, e.g., Hammerstein [5]) has the form

$$
u(x)+\int_{\Omega} k(x, y) f(y, u(y)) d y=h(x),
$$

where $d y$ is a $\sigma$-finite measure on $\Omega$; the kernel $k$ is defined on $\Omega \times \Omega, f$ is a real-valued function defined on $\Omega \times \mathbb{R}$ and is, in general, nonlinear, and $h$ is a function on $\Omega$. Setting

$$
K v(\cdot):=\int_{\Omega} k(\cdot, y) v(y) d y \quad \text { on } \Omega
$$

and $F u(\cdot):=f(\cdot, u(\cdot))$ on $\Omega$, then integral equation (9) can be put in abstract operator form as follows:

$$
u+K F u=0,
$$

where, without loss of generality, we have taken $h \equiv 0$.

Interest in (9) stems mainly from the fact that several problems that arise in differential equations, for instance, elliptic boundary value problems whose linear parts possess Green's function, can, as a rule, be transformed into the form of (9).

Furthermore, equations of Hammerstein type play crucial role in the theory of optimal control systems, in automation, and in network theory (see, e.g., Dolezale [6]).

Several existence theorems for the solution of (9) have been proved by a host of distinguished mathematicians using various techniques (see, e.g., Browder and Gupta [7, 8], Chepanovich [9], and Petryshyn and Fitzpatrick [8]). In the remaining part of this section, we highlight the techniques used by Browder and Gupta [7] and Petryshyn and Fitzpatrick [8]. To do this, we first give definitions of some terms which are required in the theorems.

In the sequel, the symbol " $\rightarrow$ " denotes strong convergence while " $\rightarrow$ " denotes weak convergence.
Definition 1 (see, e.g., [7]). A mapping $A: D(A) \subset X^{*} \rightarrow X$ is said to be hemicontinuous if it is continuous from each line segment of $X^{*}$ to the weak topology of $X$. That is, $\forall u \in D(A)$, $\forall v \in X^{*}$, and $\left(t_{n}\right)_{n \geq 1} \subset \mathbb{R}^{+}$such that $t_{n} \rightarrow 0^{+}$and $u+t_{n} v \in$ $D(A)$ for $n$ sufficiently large and we have $A\left(u+t_{n} v\right) \rightarrow A(u)$.

Definition 2 (see, e.g., [7]). Let $A: X \rightarrow X^{*}$ be a bounded monotone linear mapping. $A$ is said to be anglebounded with constant $c \geq 0$ if, for all $u, v$ in $X, \mid\langle A u, v\rangle-$ $\langle A v, u\rangle \mid \leq 2 c\{\langle A u, u\rangle\}^{1 / 2}\{\langle A v, v\rangle\}^{1 / 2}$. (This is well defined since $\langle A u, u\rangle \geq 0$ and $\langle A v, v\rangle \geq 0$ by the linearity and monotonicity of $A$.)

In [7] Browder and Gupta proved the following theorem.

Theorem 3 (Browder-Gupta [7]). Let $X$ be a real Banach space and $X^{*}$ its conjugate dual space. Let $K$ be a monotone angle-bounded continuous linear mapping of $X$ into $X^{*}$ with constant of angle-boundedness $c \geq 0$. Let $F$ be a hemicontinuous (possibly nonlinear) mapping of $X^{*}$ into $X$ such that, for a given constant $k \geq 0$,

$$
\left\langle v_{1}-v_{2}, F v_{1}-F v_{2}\right\rangle \geq-k\left\|v_{1}-v_{2}\right\|_{X^{*}}^{2}
$$

for all $v_{1}$ and $v_{2}$ in $X^{*}$. Suppose finally that there exists a constant $R$ with $k\left(1+c^{2}\right) R<1$ such that for $u$ in $X$

$$
\langle K u, u\rangle \leq R\|u\|_{X}^{2} .
$$

Then, there exists exactly one solution $w$ in $X^{*}$ of the nonlinear equation

$$
w+K F w=0 .
$$

The main tool used by the authors in proving Theorem 3 is that of splitting the linear operator $K$ via a Hilbert space and then applying a deep result of Minty [10]. Precisely, they proved that if $X$ is a real Banach space, $X^{*}$ is its dual space, and $K$ is a bounded linear mapping of $X$ into $X^{*}$ which is monotone and angle-bounded, then there exist a Hilbert space $H$, a continuous linear mapping $S$ of $X$ into $H$ with adjoint $S^{*}$ injective, and a bounded skew-symmetric linear mapping $B$ of $H$ into $H$ such that

$$
K=S^{*}(I+B) S
$$

(see Figure 1).

This factorization enabled the authors to transform the problem into another problem in a Hilbert space such that Hammerstein equation (11) has a solution if and only if the new problem has a solution in a real Hilbert space. They set $f=(I+B)^{-1}+K F K^{*}, D:=B(0,1)$, the closed unit ball in $H$, and showed that $f$ is hemicontinuous and monotone and satisfies $\langle u, f(u)\rangle \geq 0 \forall u \in D$. With these facts, they used the following result of Minty [10] to prove Theorem 3 (see [10] for definitions of terms).

Theorem 4 (Minty [10]). Let $D \subset X$ be bounded and surround 0 ; let $C \subset X$ contain $\overline{\mathrm{CO}}(D)$ and surround every point of $\overline{\mathrm{co}}(D)$ densely. Let

$$
f: C \longrightarrow X^{*}
$$




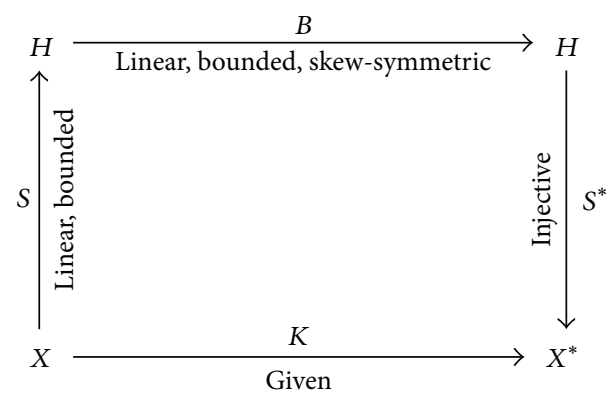

FIGURE 1: Factorization of operator, $K$.

be monotone and hemicontinuous at every point of $\overline{\mathrm{co}}(D)$ and suppose

$$
u \in D \text { implies }\langle u, f(u)\rangle \geq 0 \text {. }
$$

Then, there exists $u \in \overline{\mathrm{co}}(D)$ such that $f(u)=0$.

Petryshyn and Fitzpatrick employed deep variational techniques to prove the existence of a solution to (11). They proved the following theorems.

Theorem 5 (Petryshyn-Fitzpatrick [8]). Let $X$ be a reflexive Banach space and let $K$ be a linear, monotone, and symmetric mapping of $X$ into $X^{*}$. Suppose $f$ is a weakly (sequential) lower semicontinuous functional on $X^{*}$ such that

$$
f(u) \geq-\frac{1}{2} a_{1}\|u\|^{2}-a_{2}\|u\|^{\delta}-a_{3},
$$

where $a_{1}\|K\|<1, a_{2}>0, a_{3}>0$, and $0<\delta<2$. Suppose also that $F: X^{*} \rightarrow X$ is such that $\operatorname{grad}(f)=F$. Then,

$$
w+K F w=0
$$

has a solution in $X^{*}$.

Theorem 6 (Petryshyn-Fitzpatrick [8]). Let $X$ be a reflexive Banach space with $K: X \rightarrow X^{*}$ linear, monotone, and symmetric. Let $F: X^{*} \rightarrow X$ be potential and have a Gâteaux derivative which satisfies the inequality

$$
D F(u, v, v) \geq-a\|v\|^{2} \quad\left(v, u \in X^{*}\right)
$$

and $\operatorname{DN}(t u, v, v)$ is continuous in $t \in[0,1]$ for $u$ and $v$ fixed, where $a\|K\|<1$. Then, (19) has a solution in $X^{*}$.

In this paper, we introduce a new method, perhaps simpler than methods used so far in the literature, of proving existence of solutions of Hammerstein equation in certain cases. To achieve this, we recast (11) into a fixed point problem and use a technique recently introduced by Chidume and Zegeye [11], some existence results of Deimling [12] for zeros of accretive maps, and some surjectivity results of Browder [13] for Lipschitz strongly accretive maps. No linearity assumption is imposed on any of our maps.

\section{Preliminaries}

Let $X$ be a normed linear space and let $K$ be a convex subset of $X$. For $x \in X$, the inward set, $I_{k}(x)$, of $x$ relative to $K$, is defined as follows:

$$
I_{K}(x)=\{x+c(u-x): c \geq 1, u \in K\} .
$$

A mapping $T: K \rightarrow X$ is said to be inward if $T x \in I_{K}(x)$ for each $x \in K$ and weakly inward if Tx belongs to the closure of $I_{K}(x)$ for each $x \in K$.

A relationship between the weak inward condition and the condition

$$
\lim _{\lambda \rightarrow 0^{+}} \frac{\operatorname{dist}(x-\lambda A x, D(A))}{\lambda}=0 \quad \forall x \in D(A)
$$

for a map $A: D(A) \subset X \rightarrow X$ is given in Lemma 11. Further relationship between condition (22), the weak inward condition, and Lemma 11 can be found in [14].

In the sequel, $X$ is a $q$-uniformly smooth real Banach space, $q>1$, and $E:=X \times X$ with

$$
\|[u, v]\|_{E}=\left(\|u\|^{q}+\|v\|^{q}\right)^{1 / q} \quad \forall[u, v] \in E .
$$

If $X(=H)$ is a real Hilbert space, we will denote $E$ by $E^{H}:=$ $H \times H$.

If $F$ and $K$ are maps from $X$ to $X$ such that range of $F$ is contained in domain of $K$, that is, $R(F) \subseteq D(K)$, Chidume and Zegeye [11] defined a map $A: E \rightarrow E$ as follows:

$$
A[u, v]=[F u-v, K v+u]
$$

for all $u, v \in X$ and observed that $A[u, v]=0$ if and only if

$$
\begin{aligned}
& F u-v=0, \\
& K v+u=0,
\end{aligned}
$$

so that $u$ solves (11). System (25) can be recast as a fixed point problem as follows:

$$
\left(\begin{array}{l}
u \\
v
\end{array}\right)=\left(\begin{array}{cc}
0 & -K \\
F & 0
\end{array}\right)\left(\begin{array}{l}
u \\
v
\end{array}\right) .
$$

We will use the ideas of map $A$ on $E$.

In Lemmas 9 and 10, we use the following variant definition of accretive maps as given by Deimling [12].

Definition 7 (accretive map in the sense of Deimling [12]). Let $X$ be real Banach space. A map $A: D(A) \subset X \rightarrow X$ is said to be accretive (in the sense of Deimling) if

$$
\langle A(x)-A(y), x-y\rangle_{+} \geq 0 \quad \forall x, y \in D(A),
$$

where

$$
\langle x, y\rangle_{+}:=\sup _{j(y) \in J(y)}\langle x, j(y)\rangle, \quad \forall x, y \in X .
$$

It is evident that, in any real Banach space, an accretive map is also accretive in the sense of Deimling. The converse is true in any real Banach $X$ whose dual $X^{*}$ is strictly convex or whose normalized duality map is single-valued. This is certainly the case when $X$ is $q$-uniformly smooth, $q>1$. 
Definition 8 (see, e.g., [15]). A bounded convex subset $K$ of a Banach space $X$ is said to have normal structure if every convex subset $C$ of $K$ having more than one element contains at least one nondiametral point; that is, there exists $x^{0} \in C$ such that

$$
\begin{aligned}
& \sup \left\{\left\|x^{0}-x\right\|: x \in C\right\} \\
& \quad<\sup \{\|x-y\|: x, y \in C\}=d(C) .
\end{aligned}
$$

The Banach space $X$ is said to have normal structure if every bounded convex subset of $X$ has normal structure.

Lemma 9 (Deimling [12]). Let $X$ be a reflexive real Banach space with normal structure and let $D$ be a closed convex bounded subset of $X$. Let $A: D \rightarrow X$ be a Lipschitz and accretive map satisfying condition (22). Then, $0 \in A(D)$.

Lemma 10 (Deimling [12]). Let $X$ be real Banach space and let $D$ be a closed convex subset of $X$. Let $A: D \subset X \rightarrow X$ be an accretive continuous map such that $\langle A x, x\rangle_{+} \geq 0$ for all $x \in X$ with $\|x\| \geq R$ for some $R>0$ or $\lim \|A x\|=\infty$ as $\|x\| \rightarrow \infty$. Suppose A satisfies condition (22) and suppose that $A(D)$ is closed. Then, $0 \in A(D)$.

Lemma 11 (Caristi [16]). Let D be a convex subset of a normed linear space $X$ and let $A: D \rightarrow X$ be a map. Then condition (22) holds if and only if $(I-A)$ is weakly inward and $I$ is the identity map on D.

Remark 12. In view of Lemma 11, if $D=H$ in Lemma 10, then condition (22) can be dropped.

Lemma 13 (Xu [2]). Let $q>1$ and $E$ a smooth real Banach space. Then the following are equivalent.

(i) E is q-uniformly smooth.

(ii) There exists a constant $d_{q}>0$ such that, for all $x, y \in E$,

$$
\|x+y\|^{q} \leq\|x\|^{q}+q\left\langle y, j_{q}(x)\right\rangle+d_{q}\|y\|^{q} .
$$

(iii) There exists a constant $c_{q}>0$ such that for all $x, y \in E$ and $\lambda \in[0,1]$

$$
\begin{array}{r}
\|(1-\lambda) x+\lambda y\|^{q} \geq \\
(1-\lambda)\|x\|^{q}+\lambda\|y\|^{q} \\
-w_{q}(\lambda) c_{q}\|x-y\|^{q},
\end{array}
$$

where $w_{q}(\lambda):=\lambda^{q}(1-\lambda)+\lambda(1-\lambda)^{q}$.

From now on, $c_{q}$ and $d_{q}$ denote the constants appearing in Lemma 13.

Lemma 14 (Chidume [15], p. 173). Let $X$ be a q-uniformly smooth real Banach space. Let $F, K: X \rightarrow X$ be maps with $F$ surjective such that the following conditions hold:

(i) there exists $\alpha>0$ such that, for each $u_{1}, u_{2} \in D(F)$,

$$
\left\langle F u_{1}-F u_{2}, j_{q}\left(u_{1}-u_{2}\right)\right\rangle \geq \alpha\left\|u_{1}-u_{2}\right\|^{q}
$$

(ii) there exists $\beta>0$ such that, for each $u_{1}, u_{2} \in D(K)$,

$$
\left\langle K u_{1}-K u_{2}, j_{q}\left(u_{1}-u_{2}\right)\right\rangle \geq \beta\left\|u_{1}-u_{2}\right\|^{q}
$$

(iii) $\left(1+d_{q}\right)\left(1+c_{q}\right) \geq 2^{q}, \min \{\alpha, \beta\}=: \gamma>\left(\left(1+d_{q}\right)(1+\right.$ $\left.\left.c_{q}\right)-2^{q}\right) / q\left(1+c_{q}\right)$.

Let a map $A: E \rightarrow E$ be defined by (24). Then, for each $z_{1}$, $z_{2} \in E$,

$$
\begin{aligned}
& \left\langle A z_{1}-A z_{2}, j_{q}\left(z_{1}-z_{2}\right)\right\rangle \\
& \quad \geq\left[\gamma-q^{-1}\left(\left(1+d_{q}\right)-\frac{2^{q}}{\left(1+c_{q}\right)}\right)\right]\left\|z_{1}-z_{2}\right\|^{q} .
\end{aligned}
$$

Lemma 15. Let $H$ be a real Hilbert space. Let $K: D(K) \subset$ $H \rightarrow H, F: D(F) \subset H: \rightarrow H$ be two monotone maps such that $R(F) \subset D(K)$. Then the map $A: D(F) \times D(K) \subset E^{H} \rightarrow$ $E^{H}$ defined by (24) is monotone.

Proof. The proof follows from the lines of argument of the proof of Lemma 14 (see Chidume and Zegeye [11]).

Lemma 16 (Chidume [15], p. 173). Let $X$ be a q-uniformly smooth real Banach space and let $K: D(K) \subset X \rightarrow X$, $F: D(F) \subset X \rightarrow X$ be two Lipschitz maps such that $R(F) \subset D(K)$. Let $A: D(A) \subset E$ be a map such that $D(F) \times D(K)=D(A)$ and defined by (24). Then, $A$ is Lipschitz.

We need the following definition which was given by Browder [17].

Definition 17 (Browder [17]). Let $X$ and $Y$ be real Banach spaces with $Y^{*}$ the conjugate space of $Y$. Let $\phi$ be a mapping of $X$ into $Y^{*}$ such that $\phi(X)$ is dense in $Y^{*}$ with

$$
\begin{aligned}
\|\phi(x)\|_{Y^{*}} & =\|x\|, \\
\phi(\xi x) & =\xi \phi(x)
\end{aligned}
$$

for all $x \in X, \xi \geq 0$. The mapping $f: X \rightarrow Y$ is said to be strongly $\phi$-accretive if there exists $c>0$ such that, for all $x$ and $u$ in $X$,

$$
\langle f(x)-f(u), \phi(x-u)\rangle \geq c\|x-u\|^{2} .
$$

It follows from this definition that if $X$ is a real Banach space such that the normalized duality map $J$ is single-valued and $J(X)$ is dense in $X^{*}$ (e.g., when $X$ is a reflexive and smooth real Banach space), then a strongly accretive map $A: X \rightarrow X$ is $J$-strongly accretive.

Theorem 18 (Browder [13]). Let $X$ and $Y$ be Banach spaces with $Y^{*}$ uniformly convex and suppose $f: X \rightarrow Y$ is a strongly $\phi$-accretive mapping satisfying a Lipschitz condition on each bounded subset of $X$. Then, $f(X)=Y$.

The following corollary follows from Theorem 18. 
Corollary 19. Let $X$ be a real Banach space with uniformly convex dual $X^{*}$ and suppose $f: X \rightarrow X$ is a strongly accretive Lipschitz mapping. Then, $f(X)=X$.

\section{Main Results}

Let $X:=L_{p}, 1<p<2$, and let $E:=X \times X$ with $\|z\|_{E}^{2}:=$ $\|[u, v]\|_{E}^{2}=\|u\|_{X}^{2}+\|v\|_{E}^{2}$ for arbitrary $z=[u, v] \in E$. For $L_{p}$ spaces, $1<p<2$, the following estimate has been established (see, e.g., Chidume [15], p. 183):

$$
\begin{aligned}
& A\left(u_{1}, u_{2}, v_{1}, v_{2}\right) \\
& \quad:=\left[\left\langle v_{1}-v_{2}, j\left(u_{1}-u_{2}\right)\right\rangle+\left\langle u_{1}-u_{2}, j\left(u_{2}-u_{1}\right)\right\rangle\right] \\
& \quad \leq p(2-p)\left(\left\|u_{1}-u_{2}\right\|^{2}+\left\|v_{1}-v_{2}\right\|^{2}\right)
\end{aligned}
$$

$$
\forall u_{1}, u_{2}, v_{1}, v_{2} \in X
$$

We begin with a proof of the following theorem for $L_{p}$ spaces, $1<p<2$, which is new.

Theorem 20. Let $X=L_{p}(1<p<2)$; let $F, K: X \rightarrow X$ be mappings such that $D(K)=F(X)=X$ and the following conditions hold:

(a) there exists $\alpha>0$ such that, for each $u_{1}, u_{2} \in X$,

$$
\left\langle F u_{1}-F u_{2}, j\left(u_{1}-u_{2}\right)\right\rangle \geq \alpha\left\|u_{1}-u_{2}\right\|^{2}
$$

(b) there exists $\beta>0$ such that, for each $u_{1}, u_{2} \in X$,

$$
\left\langle K u_{1}-K u_{2}, j\left(u_{1}-u_{2}\right)\right\rangle \geq \beta\left\|u_{1}-u_{2}\right\|^{2}
$$

(c) $\gamma:=\min \{\alpha, \beta\}$ with $\gamma>p(2-p)$.

Let $E:=X \times X$ and define $A: E \rightarrow E$ by (24) for all $[u, v] \in E$. Then, for arbitrary $z_{1}, z_{2} \in E$, the following inequality holds:

$$
\begin{aligned}
& \left\langle A z_{1}-A z_{2}, j^{E}\left(z_{1}-z_{2}\right)\right\rangle \\
& \quad \geq[\gamma-p(2-p)]\left\|z_{1}-z_{2}\right\|^{2} .
\end{aligned}
$$

Proof. We compute as follows:

$$
\begin{aligned}
\left\langle A z_{1}-A z_{2}, j^{E}\left(z_{1}-z_{2}\right)\right\rangle & \\
= & \left\langle F u_{1}-F u_{2}, j\left(u_{1}-u_{2}\right)\right\rangle-\left\langle v_{1}-v_{2}, j\left(u_{1}-u_{2}\right)\right\rangle \\
& +\left\langle K v_{1}-K v_{2}, j\left(v_{1}-v_{2}\right)\right\rangle \\
& +\left\langle u_{1}-u_{2}, j\left(v_{1}-v_{2}\right)\right\rangle \\
\geq & \alpha\left\|u_{1}-u_{2}\right\|^{2}+\beta\left\|v_{1}-v_{2}\right\|^{2} \\
& -\left\langle v_{1}-v_{2}, j\left(u_{1}-u_{2}\right)\right\rangle+\left\langle u_{1}-u_{2}, j\left(v_{1}-v_{2}\right)\right\rangle \\
\geq & \gamma\left(\left\|u_{1}-u_{2}\right\|^{2}+\left\|v_{1}-v_{2}\right\|^{2}\right) \\
& -\left[\left\langle v_{1}-v_{2}, j\left(u_{1}-u_{2}\right)\right\rangle-\left\langle u_{1}-u_{2}, j\left(v_{1}-v_{2}\right)\right\rangle\right]
\end{aligned}
$$

$$
\begin{aligned}
\geq & \gamma\left\|z_{1}-z_{2}\right\|^{2}-A\left(u_{1}, u_{2}, v_{1}, v_{2}\right) \\
\geq & \gamma\left\|z_{1}-z_{2}\right\|^{2} \\
& -p(2-p)\left(\left\|u_{1}-u_{2}\right\|^{2}+\left\|v_{1}-v_{2}\right\|^{2}\right) \\
= & (\gamma-p(2-p))\left\|z_{1}-z_{2}\right\|^{2} \quad \forall z_{1}, z_{2} \in E,
\end{aligned}
$$

completing proof of the theorem.

Remark 21. Observe that the condition $1+\sqrt{1-\gamma}<p<2$ implies $\gamma>p(2-p)$.

We now prove the following existence theorems.

\subsection{The Case of Hilbert Spaces}

Theorem 22. Let $H$ be a real Hilbert space and let $K$ : $D(K) \subset H \rightarrow H, F: D(F) \subset H \rightarrow H$ be two Lipschitz monotone maps such that $D(F)$ and $D(K)$ are closed, convex, and bounded and $R(F) \subset D(K)$. Let $A: D(A) \subset E^{H} \rightarrow$ $E^{H}$ be a map such that $D(F) \times D(K)=: D(A)$ and $A$ is defined by (24). Suppose that A satisfies condition (22). Then, Hammerstein equation (11) has a solution.

Proof. The fact that $K$ and $F$ are Lipschitz and monotone implies that $A$ is Lipschitz and monotone (Lemmas 15 and 16). Since the normalized duality map is the identity map in real Hilbert spaces, monotonicity of $A$ is equivalent to accretivity in the sense of Deimling. Also $D(A)$ is closed and convex since $D(F)$ and $D(K)$ are. Therefore, by Lemma 9, $0 \in A(D)$; that is, there exists $[u, v] \in D$ such that $F u-v=0$ and $K v+u=0$. So $u$ solves (11). This completes the proof.

Theorem 23. Let $H$ be a real Hilbert space and let $K: D(K) \subset$ $H \rightarrow H, F: D(F) \subset H \rightarrow H$ be two continuous monotone maps such that $D(F)$ and $D(K)$ are closed and convex and $R(F) \subset D(K)$. Let $A: D(A) \subset E^{H} \rightarrow E^{H}$ be a map such that $D(F) \times D(K)=: D(A)$ and $A$ is defined by (24). Suppose that $\langle A w, w\rangle \geq 0$ for all $w \in D(A)$ with $\|w\| \geq R$ for some $R>0$ or $\lim \|A w\|=\infty$ as $\|w\| \rightarrow \infty$ and suppose that $A$ satisfies condition (22). Suppose that $A(D(A))$ is closed. Then, Hammerstein equation (11) has a solution.

Proof. The fact that $K$ and $F$ are monotone implies that $A$ is monotone (Lemma 15). The fact that $D(F)$ and $D(K)$ are closed and convex implies that $D(A)$ is closed and convex. Also since $E^{H}$ is a real Hilbert space and the normalized duality map of any real Hilbert space is the identity map, we have $\langle A w, w\rangle_{+}=\langle A w, w\rangle$ for all $w \in D(A)$. Therefore, the assumptions on $A$ and $D(A)$ together with Lemma 10 give that $0 \in A(D)$; that is, there exists $[u, v] \in D$ such that $F u-v=0$ and $K v+u=0$. So $u$ solves (11). This completes the proof.

Corollary 24. Let $H$ be a real Hilbert space and let $K, F$ : $H \rightarrow H$ be two continuous monotone maps defined on $H$. Let $A: E^{H} \rightarrow E^{H}$ be a map defined by (24). Suppose that 
$\langle A w, w\rangle \geq 0$ for all $w \in E^{H}$ with $\|w\| \geq R$ for some $R>0$ or $\lim \|A w\|=\infty$ as $\|w\| \rightarrow \infty$. Suppose that $A\left(E^{H}\right)$ is closed. Then, Hammerstein equation (11) has a solution.

Proof. Since $A$ is defined on $E^{H}$, it satisfies condition (22). Therefore, the result follows from Theorem 23.

\subsection{The Case of $L^{p}$ Spaces, $1<p<\infty$}

Theorem 25. Let $K: D(K) \subset L^{p} \rightarrow L^{p}$ and $F: D(F) \subset$ $L^{p} \rightarrow L^{p}$ be two Lipschitz mappings satisfying the following conditions:

(a) there exists $\alpha>0$ such that, for each $u_{1}, u_{2} \in D(F)$,

$$
\left\langle F u_{1}-F u_{2}, j\left(u_{1}-u_{2}\right)\right\rangle \geq \alpha\left\|u_{1}-u_{2}\right\|^{2} ;
$$

(b) there exists $\beta>0$ such that, for each $u_{1}, u_{2} \in D(K)$,

$$
\left\langle K u_{1}-K u_{2}, j\left(u_{1}-u_{2}\right)\right\rangle \geq \beta\left\|u_{1}-u_{2}\right\|^{2} \text {. }
$$

Let $D(F)$ and $D(K)$ be closed, convex, and bounded such that $R(F) \subset D(K)$. Let $E:=L^{p} \times L^{p}$ and let $A: D(A) \subset E \rightarrow E$ be a map such that $D(F) \times D(K)=: D(A)$ and $A$ is defined by (24). Suppose that $A$ satisfies condition (22). Let $\gamma:=\min \{\alpha, \beta\}$. If $2 \leq p<\gamma+\sqrt{\gamma^{2}+4}$ or $1+\sqrt{1-\gamma}<p \leq 2$, then Hammerstein equation (11) has a solution.

Proof. The fact that $K$ and $F$ are Lipschitz implies that $A$ is Lipschitz by Lemma 16 . Also $D(A)$ is closed and convex since $D(F)$ and $D(K)$ are.

Case $1\left(2 \leq p<\gamma+\sqrt{\gamma^{2}+4}\right)$. In this case $L^{p}$ is 2 -uniformly smooth space and $c_{q}=d_{q}=p-1$ (see, e.g., [2]). Therefore, $\left(1+c_{q}\right)\left(1+d_{q}\right)=p^{2} \geq 4=2^{q}$ and

$$
\gamma>\frac{1}{2 p}\left(p^{2}-4\right)=\frac{\left(1+d_{q}\right)\left(1+c_{q}\right)-2^{q}}{q\left(1+c_{q}\right)}
$$

for $2 \leq p<\gamma+\sqrt{\gamma^{2}+4}$. This implies by Lemma 14 that $A$ is accretive. Therefore, $A$ is accretive in the sense of Deimling. Hence, using Lemma 9, we have that $0 \in A(D)$; that is, there exists $[u, v] \in D$ such that $F u-v=0$ and $K v+u=0$. So $u$ solves (11).

Case $2(1+\sqrt{1-\gamma}<p \leq 2)$. The condition $1+\sqrt{1-\gamma}<$ $p \leq 2$ implies that $\gamma>p(2-p)$. Hence, by Theorem $20, A$ is accretive. We conclude as in Case 1. This completes the proof.

Theorem 26. Let $K: D(K) \subset L^{p} \rightarrow L^{p}, F: D(F) \subset$ $L^{p} \rightarrow L^{p}$ be two continuous mappings satisfying the following conditions:

(a) there exists $\alpha>0$ such that, for each $u_{1}, u_{2} \in D(F)$,

$$
\left\langle F u_{1}-F u_{2}, j\left(u_{1}-u_{2}\right)\right\rangle \geq \alpha\left\|u_{1}-u_{2}\right\|^{2} ;
$$

(b) there exists $\beta>0$ such that, for each $u_{1}, u_{2} \in D(K)$,

$$
\left\langle K u_{1}-K u_{2}, j\left(u_{1}-u_{2}\right)\right\rangle \geq \beta\left\|u_{1}-u_{2}\right\|^{2} .
$$

Let $D(F)$ and $D(K)$ be closed and convex, such that $R(F) \subset$ $D(K)$. Let $E:=L^{p} \times L^{p}$ and let $A: D(A) \subset E \rightarrow E$ be a mapping such that $D(F) \times D(K)=: D(A)$ and $A$ is defined by (24) for $[u, v] \in D(A)$. Suppose that $\langle A w, w\rangle_{+} \geq 0$ for all $w \in D(A)$ with $\|w\| \geq R$ for some $R>0$ or $\lim \|A w\|=\infty$ as $\|w\| \rightarrow \infty$ and suppose A satisfies condition (22). Let $\gamma:=$ $\min \{\alpha, \beta\}$. If $2 \leq p<\gamma+\sqrt{\gamma^{2}+4}$ or $1+\sqrt{1-\gamma}<p \leq 2$, then Hammerstein equation (11) has a solution.

Proof. Evidently, continuity of $K$ and $F$ gives the continuity of $A$. Also $D(A)$ is closed and convex since $D(F)$ and $D(K)$ are. The rest follows as in the proof of Theorem 25. This completes the proof.

Corollary 27. Let $K: D(K) \subset L^{p} \rightarrow L^{p}, F: D(F) \subset$ $L^{p} \rightarrow L^{p}$ be two continuous accretive mappings satisfying the following conditions:

(a) there exists $\alpha>0$ such that, for each $u_{1}, u_{2} \in D(F)$,

$$
\left\langle F u_{1}-F u_{2}, j\left(u_{1}-u_{2}\right)\right\rangle \geq \alpha\left\|u_{1}-u_{2}\right\|^{2}
$$

(b) there exists $\beta>0$ such that, for each $u_{1}, u_{2} \in D(K)$,

$$
\left\langle K u_{1}-K u_{2}, j\left(u_{1}-u_{2}\right)\right\rangle \geq \beta\left\|u_{1}-u_{2}\right\|^{2} \text {. }
$$

Let $D(F)=L^{p}=D(K)$. Let $E:=L^{p} \times L^{p}$ and let $A: E \rightarrow$ $E$ be a mapping defined by (24) $[u, v] \in D(A)$. Suppose that $\langle A w, w\rangle_{+} \geq 0$ for all $w \in E$ with $\|w\| \geq R$ for some $R>0$ or $\lim \|A w\|=\infty$ as $\|w\| \rightarrow \infty$. Let $\gamma:=\min \{\alpha, \beta\}$. If $2 \leq$ $p<\gamma+\sqrt{\gamma^{2}+4}$ or $1+\sqrt{1-\gamma}<p \leq 2$, then Hammerstein equation (11) has a solution.

Proof. Since $A$ is defined on $E$, it satisfies condition (22) of Theorem 26. Also $D(A)$ is closed and convex. Therefore, the result follows from Theorem 26.

\subsection{The Case of Hilbert Spaces with Lipschitz Strongly Monotone Mappings}

Theorem 28. Let $H$ be a real Hilbert space and let $K: H \rightarrow$ $H, F: H \rightarrow H$ be two Lipschitz strongly monotone mappings with constants $\alpha, \beta$, respectively. Let $A: E^{H} \rightarrow E^{H}$ be a mapping defined by (24) for $[u, v] \in E^{H}$. Then, Hammerstein equation (11) has a solution.

Proof. Using Lemma 16 we have that $A$ is Lipschitz. Also since every real Hilbert space is $q$-uniformly smooth with $q=2$, $d_{q}=c_{q}=1$, we have that $\left(1+c_{q}\right)\left(1+d_{q}\right)=4=2^{q}$. Also $\min \{\alpha, \beta\}>0=\left(\left(1+c_{q}\right)\left(1+d_{q}\right)-2^{q}\right) / q$. Therefore, $A$ is strongly monotone by Lemma 14 . Since $E^{H}$ is a real Hilbert space and every real Hilbert space is uniformly convex, we invoke Corollary 19 to obtain that $A\left(E^{H}\right)=E^{H}$. So there 
exists $[u, v] \in E^{H}$ such that $A[u, v]=0$; that is, $F u-v=$ $0, K v+u=0$. Hence $u$ solves (11). This completes the proof.

3.4. The Case of $L_{p}$ Spaces, $1<p<\infty$, with Lipschitz Strongly Accretive Mappings

Theorem 29. Let $K: L^{p} \rightarrow L_{p}, F: L^{p} \rightarrow L_{p}$ be two Lipschitz mappings satisfying the following conditions:

(a) there exists $\alpha>0$ such that, for each $u_{1}, u_{2} \in L^{p}$,

$$
\left\langle F u_{1}-F u_{2}, j\left(u_{1}-u_{2}\right)\right\rangle \geq \alpha\left\|u_{1}-u_{2}\right\|^{2} ;
$$

(b) there exists $\beta>0$ such that, for each $u_{1}, u_{2} \in L^{p}$,

$$
\left\langle K u_{1}-K u_{2}, j\left(u_{1}-u_{2}\right)\right\rangle \geq \beta\left\|u_{1}-u_{2}\right\|^{2} .
$$

Let $E:=L^{p} \times L^{p}$ and let $A: E \rightarrow E$ be a mapping defined by (24). Let $\gamma:=\min \{\alpha, \beta\}$. If $2 \leq p<\gamma+\sqrt{\gamma^{2}+4}$ or $1+\sqrt{1-\gamma}<$ $p \leq 2$, then, Hammerstein equation (11) has a solution.

Proof. Using Lemma 16 we have that $A$ is Lipschitz.

Case $1\left(2 \leq p<\gamma+\sqrt{\gamma^{2}+4}\right)$. In this case $L^{p}$ is 2 -uniformly smooth space and $c_{q}=d_{q}=p-1$ (see, e.g., [2]). Therefore, $\left(1+c_{q}\right)\left(1+d_{q}\right)=p^{2} \geq 4=2^{q}$ and

$$
\gamma>\frac{1}{2 p}\left(p^{2}-4\right)=\frac{\left(1+d_{q}\right)\left(1+c_{q}\right)-2^{q}}{q\left(1+c_{q}\right)}
$$

for $2 \leq p<\gamma+\sqrt{\gamma^{2}+4}$. This implies by Lemma 14 that $A$ is strongly accretive. Since every $L_{p}$ space, $2 \leq p<\gamma+\sqrt{\gamma^{2}+4}$, is uniformly convex, by Corollary $19, A\left(L_{p}\right)=L_{p}$. Therefore there exists $[u, v] \in D$ such that $A[u, v]=0$; that is, $F u-v=0$ and $K v+u=0$. So $u$ solves (11).

Case $2(1+\sqrt{1-\gamma}<p \leq 2)$. The inequality $1+\sqrt{1-\gamma}<$ $p \leq 2$ implies that $\gamma>p(2-p)$. Hence by Theorem $20 A$ is strongly accretive. The result now follows as in Case 1 since every $L_{p}$ space, $1+\sqrt{1-\gamma}<p \leq 2$, is uniformly convex. This completes the proof.

\section{Conflict of Interests}

The authors declare that there is no conflict of interests regarding the publication of this paper.

\section{References}

[1] J. Lindenstrauss and L. Tzafriri, Classical Banach Spaces II: Function Space, vol. 97 of Ergebnisse der Mathematik und ihrer Grenzgebiete, Springer, Berlin, Germany, 1979.

[2] H.-K. Xu, "Inequalities in Banach spaces with applications," Nonlinear Analysis: Theory, Methods \& Applications, vol. 16, no. 12, pp. 1127-1138, 1991.
[3] I. Cioranescu, Geometry of Banach Spaces, Duality Mappings and Nonlinear Problems, Kluwer, Dordrecht, The Netherlands, 1990.

[4] S. Reich, "Geometry of Banach spaces, duality mappings and nonlinear problems, by Ioana Cioranescu," Bulletin of the American Mathematical Society, vol. 26, pp. 367-370, 1992.

[5] A. Hammerstein, "Nichtlineare integralgleichungen nebst anwendungen," Acta Mathematica, vol. 54, no. 1, pp. 117-176, 1930.

[6] V. Dolezale, Monotone Operators and Applications in Automation and Network Theory, vol. 3 of Studies in Automation and Control, Elsevier Science, New York, NY, USA, 1979.

[7] F. E. Browder and C. P. Gupta, "Monotone operators and nonlinear integral equations of Hammerstein type," Bulletin of the American Mathematical Society, vol. 75, pp. 1347-1353, 1969.

[8] W. V. Petryshyn and P. M. Fitzpatrick, "New existence theorems for nonlinear equations of Hammerstein type," Transactions of the American Mathematical Society, vol. 160, pp. 31-69, 1971.

[9] R. Sh. Chepanovich, "Nonlinear Hammerstein equations and fixed points," Publications de l'Institut Mathématique, vol. 35, no. 49, pp. 119-123, 1984.

[10] G. J. Minty, “On a 'monotonicity' method for the solution of nonlinear equations in Banach spaces," Proceedings of the National Academy of Sciences of the United States of America, vol. 50, no. 6, pp. 1038-1041, 1963.

[11] C. E. Chidume and H. Zegeye, "Iterative approximation of solutions of nonlinear equations of Hammerstein type," Abstract and Applied Analysis, vol. 2003, no. 6, pp. 353-365, 2003.

[12] K. Deimling, "Zeros of accretive operators," Manuscripta Mathematica, vol. 13, pp. 365-374, 1974.

[13] F. E. Browder, "Normal solvability and existence theorems for nonlinear mappings in Banach spaces," in Problems in NonLinear Analysis (C.I.M.E., IV Ciclo, Varenna, 1970), pp. 19-35, Edizioni Cremonese, Rome, Italy, 1971.

[14] S. Reich, "On fixed point theorems obtained from existence theorems for differential equations," Journal of Mathematical Analysis and Applications, vol. 54, no. 1, pp. 26-36, 1976.

[15] C. Chidume, Geometric Properties of Banach Spaces and Nonlinear Iterations, vol. 1965 of Lecture Notes in Mathematics, Springer, London, UK, 2009.

[16] J. Caristi, "Fixed point theorems for mappings satisfying inwardness conditions," Transactions of the American Mathematical Society, vol. 215, pp. 241-251, 1976.

[17] F. E. Browder, "Normal solvability $\varphi$-accretive mappings of Banach spaces," Bulletin of the American Mathematical Society, vol. 78, pp. 186-192, 1972. 


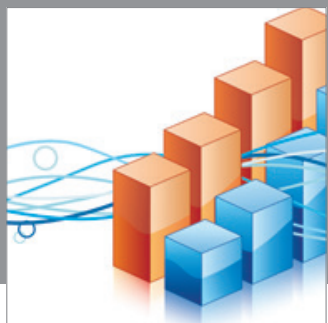

Advances in

Operations Research

mansans

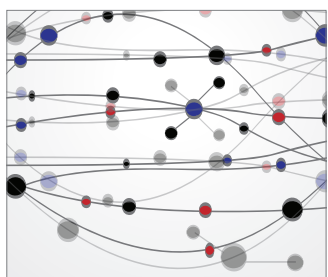

The Scientific World Journal
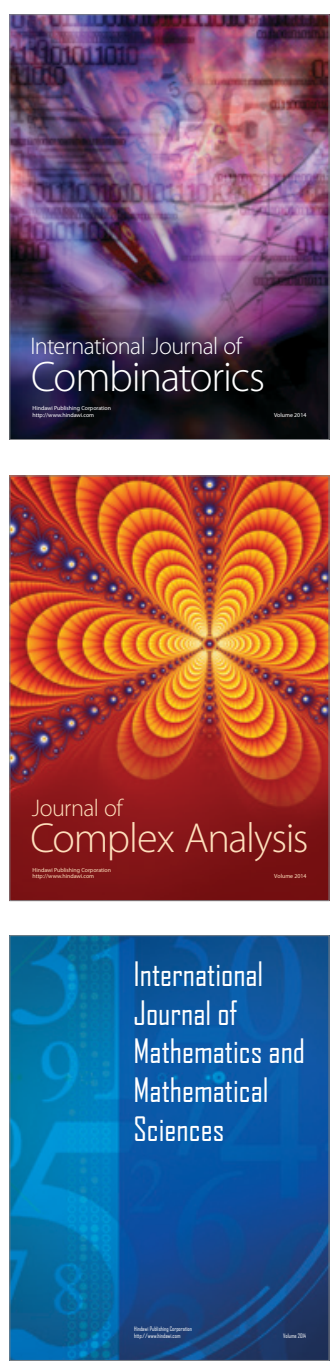
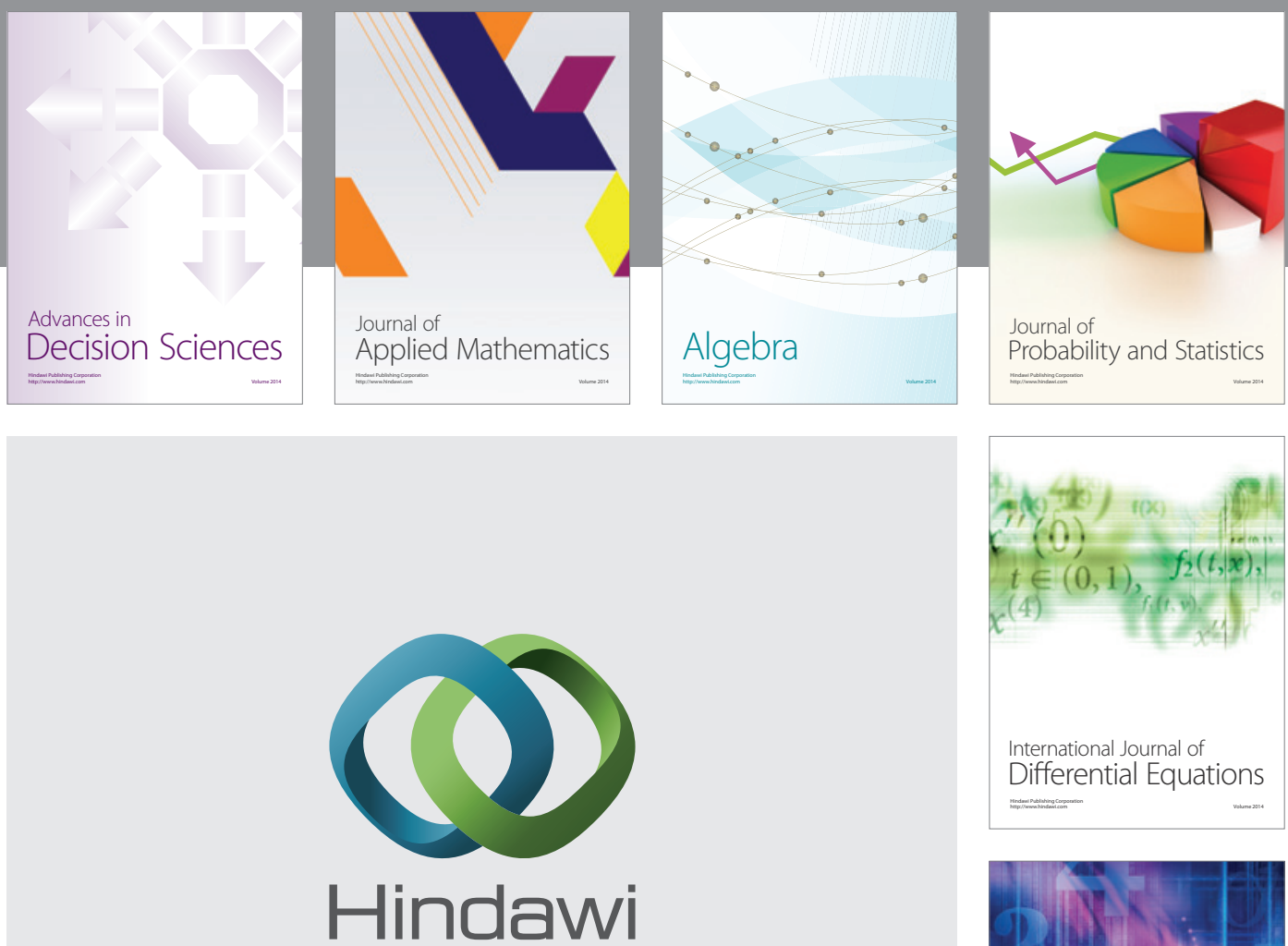

Submit your manuscripts at http://www.hindawi.com
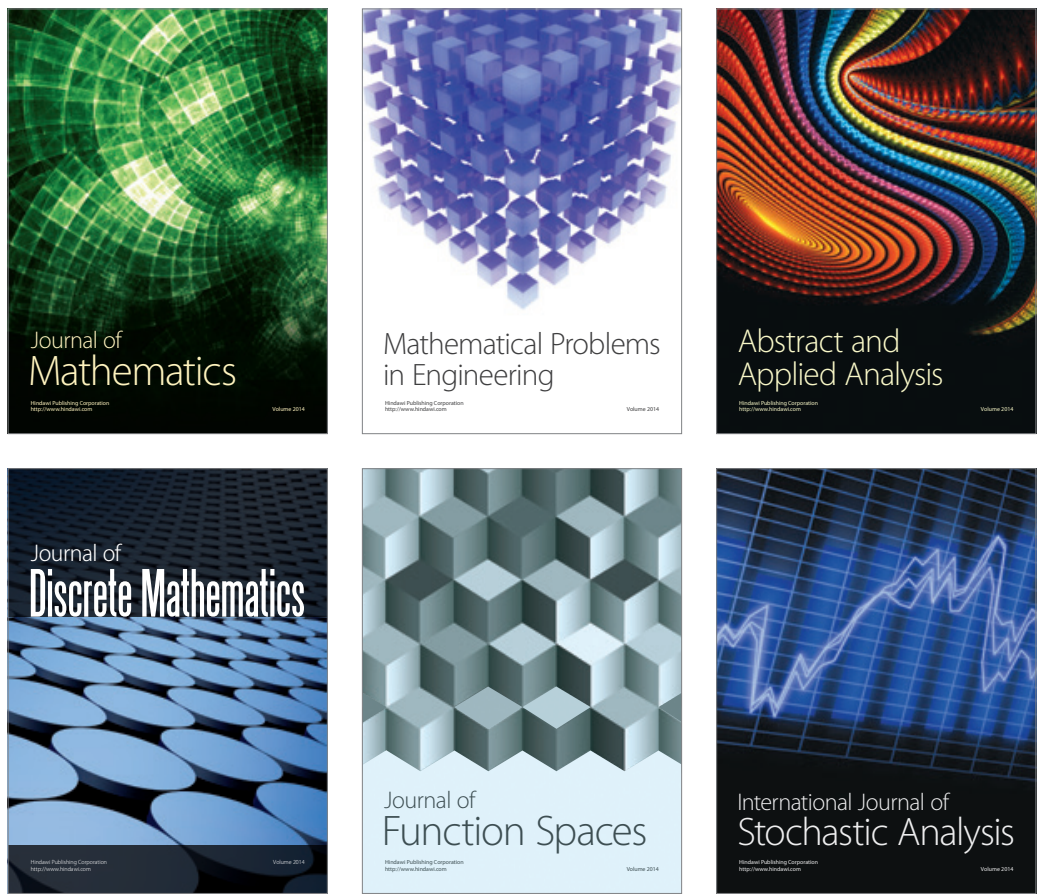

Journal of

Function Spaces

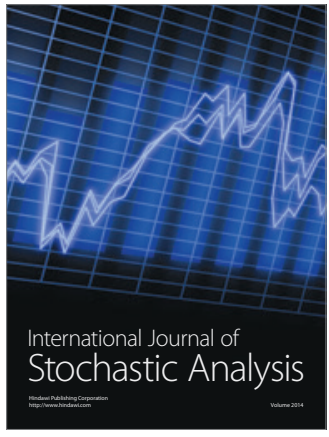

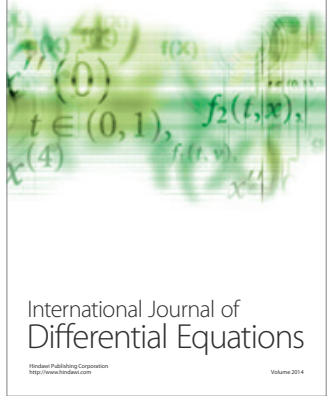
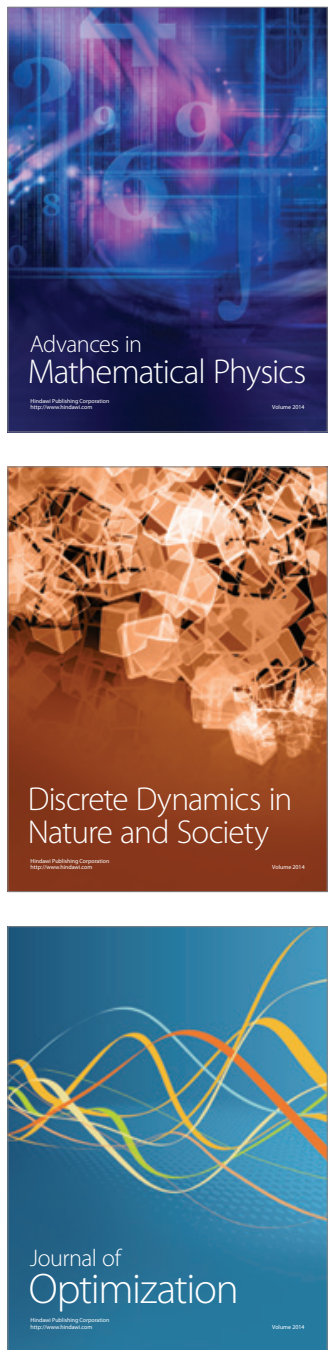